\title{
Bone versus implant: an atypical presentation of a typical complication of forearm fractures
}

\author{
Pulak Vatsya, Anupam Gupta*, Samarth Mittal, Vivek Trikha
}

Department of Orthopaedics, All India Institute of Medical Sciences, New Delhi, India

Received: 25 April 2021

Revised: 03 June 2021

Accepted: 05 June 2021

\author{
*Correspondence: \\ Dr. Anupam Gupta, \\ E-mail: anupam1054@yahoo.com
}

Copyright: ( $)$ the author(s), publisher and licensee Medip Academy. This is an open-access article distributed under the terms of the Creative Commons Attribution Non-Commercial License, which permits unrestricted non-commercial use, distribution, and reproduction in any medium, provided the original work is properly cited.

\begin{abstract}
Both bone forearm fractures are one of the most common upper limb fractures operated by orthopedicians. Although the primary surgery is usually relatively straight forward and simple, but we present a case where the primary surgery failed and the patient presented with a deformed hand after 5 months, even though there were signs of union on radiographs. When operated upon, although the ulna had undergone primary bone healing and a peri-implant fracture had occurred, whereas radius had refractured from the fracture site and the plate was bent giving the deformed appearance. We discuss this unique complication, the planning, difficulties and scope of errors in such a situation, where the race to union is won by ulna but lost by radius.
\end{abstract}

Keywords: Trauma, Implant failure, Revision surgery, Non-union, Complications, Both bone forearm

\section{INTRODUCTION}

A forerunner amongst upper limb fractures (1.35 per 10,000 population), both bone forearm fractures have long been the bread and butter surgeries for orthopedic surgeons. ${ }^{1}$ Furthermore, minimal structures at risk, good dissection learning as well as applying plates on flat surfaces, make these a playground for young orthopedic surgeons to understand and apply basic principles of fracture fixation and plating. With established standard of care being open reduction and internal fixation (ORIF) with plating, the high volume being churned out has led to a plethora of complications. From the age old complications of infection, nonunion, compartment syndrome or delayed union to more serious complications of radioulnar synostosis and refracture after implant removal, this fracture continues to entice orthopedic surgeons around the globe. ${ }^{1}$

We explore this case, wherein a young male following a standard both bone forearm fracture fixation, returned to us with a refracture. We try to find out why our fixation failed, what could be done better and lessons for future.

\section{CASE REPORT}

A 36 year male, army personnel by profession and right handed, presented to us ensuing a road traffic accident. Following standard advanced trauma life support (ATLS) protocol, the patient was diagnosed with an isolated closed left both bone forearm fracture (AO 22A3) and planned for open reduction and internal fixation (ORIF) with plating. Subsequent to general anesthesia a standard Henry's approach for radius and incision along ulnar flare were used for exposure, and both bones were fixed with titanium MonoLoc locking compression plate (Kanghui, a Medtronic company ${ }^{\mathrm{TM}}$ ). A six hole-locking compression plates (LCP) was applied on the medial surface in the ulna while a seven-hole LCP was applied volarly on the radius. Standard postoperative regimen included a single shot intravenous antibiotic and elbow range of motion exercises. With surgical wound being deemed healthy, the 
patient was discharged on post-operative day 2. Patient was in regular follow-up and showed signs of good bone healing (both the bones showed evidence of union in routine 3 monthly follow up x-rays).

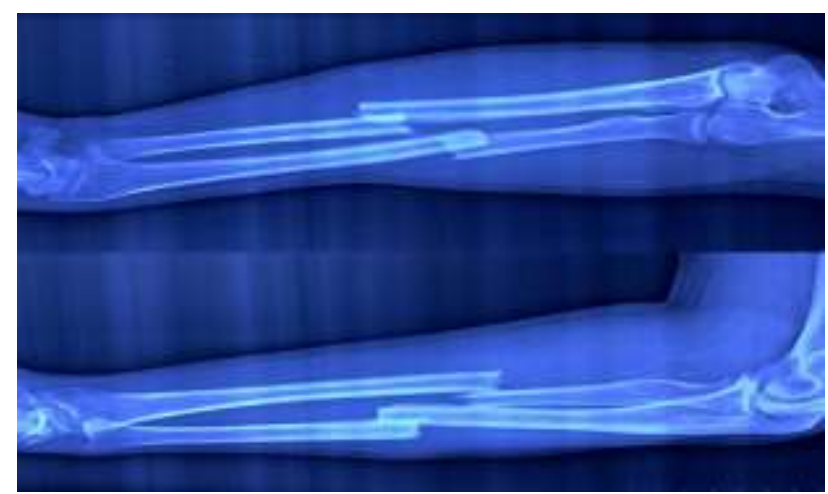

Figure 1: Preoperative radiology.

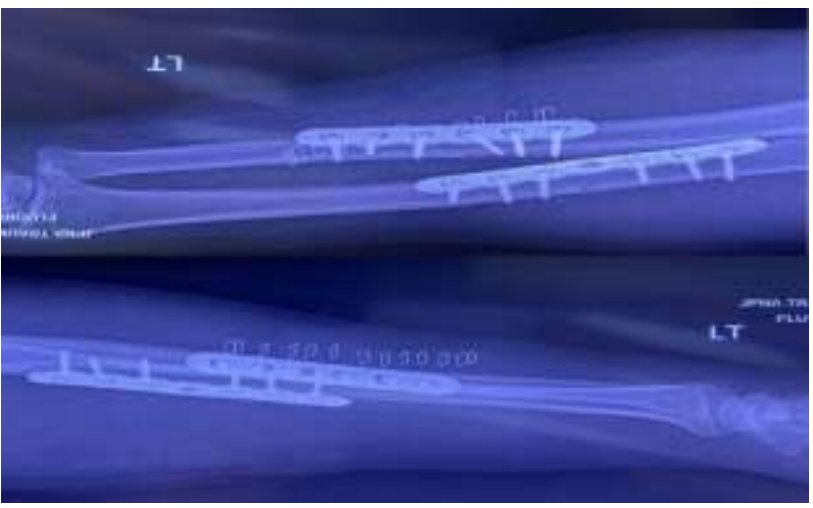

Figure 2: Post-operative x-ray after first surgery.

5 months postoperatively, the patient suffered a trivial fall and presented to us with severe pain and a deformed left forearm. Fresh radiographs revealed refracture of the radius from the old fracture site and a fracture of ulna from the terminal plate hole proximally. Work up to investigate any underlying subclinical infection which could have caused a septic nonunion was done. Inflammatory markers including erythrocyte sedimentation rate (ESR), Creactive protein (CRP), differential leucocyte count (DLC) and total leucocyte count (TLC) were within normal range. After ruling out infection, the patient was planned for implant removal and refixation with plates. Intraoperatively, using the previous incision and the same Henry's approach, the fractures were exposed. There was no evidence of any infection or unhealthy fibrous tissue. The plate on the radius had bent ( 40 degrees) as the bone had refractured and impacted at the original fracture site. On the contrary, the ulnar fracture site was completely united with primary bone healing and the fracture had occurred from the last screw hole proximally. Both the plates were removed, bone ends freshened and fractures were reduced. Ulnar plate was applied on the dorsal surface of the bone this time to avoid drilling into the same holes. Although, iliac crest was prepared for bone grafting, but the radius fracture ends appeared hypertrophic and grafting was not considered essential. Long LCP plates (AO, Synthes 10 hole each) were used to ensure adequate purchase of screws and the wound was closed in layers.

On post-operative radiographs the fracture reduction was acceptable but the ulnar plate appeared to end at the last screw hole of the previous plate distally creating a significant stress riser.

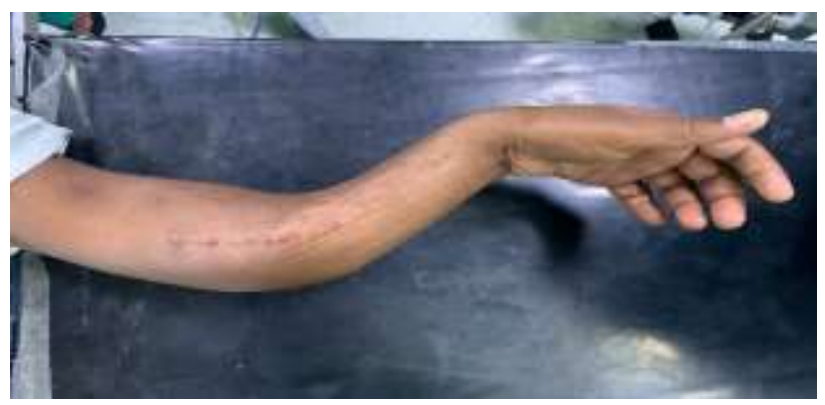

Figure 3: The deformed hand after second trauma.

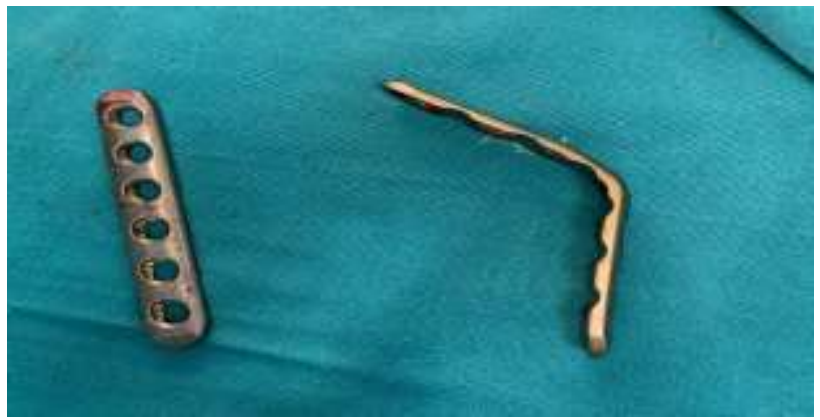

Figure 4: The deformed (but unbroken) implants (ulnar plate on left and bent radius plate on right).

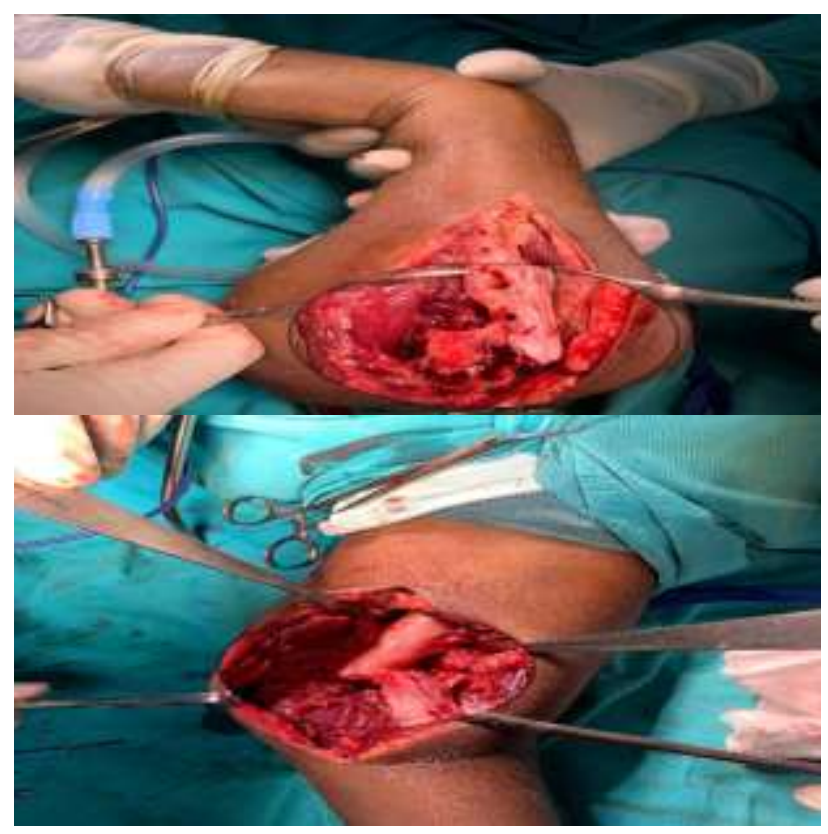

Figure 5: Ulnar refracture from the terminal screw site, where as radius refracture from the old fracture site. 
At 6 months follow up, the patient was doing excellent functionally. Full elbow and wrist range of motion (ROM) had been achieved. Wounds were healed appropriately and ulna showed evidence of bone union. Radius on the contrary still showed signs of hypertrophic callus formation but not of primary union. Also, the patient complained of inability to flex the left $1^{\text {st }}$ interphalangeal joint. On examination the patient only had motor loss but no sensory involvement. Flexor pollicis longus (FPL) function lost in the patient was suspected to be due to anterior interosseous nerve (AIN) injury as described in literature. $^{2}$

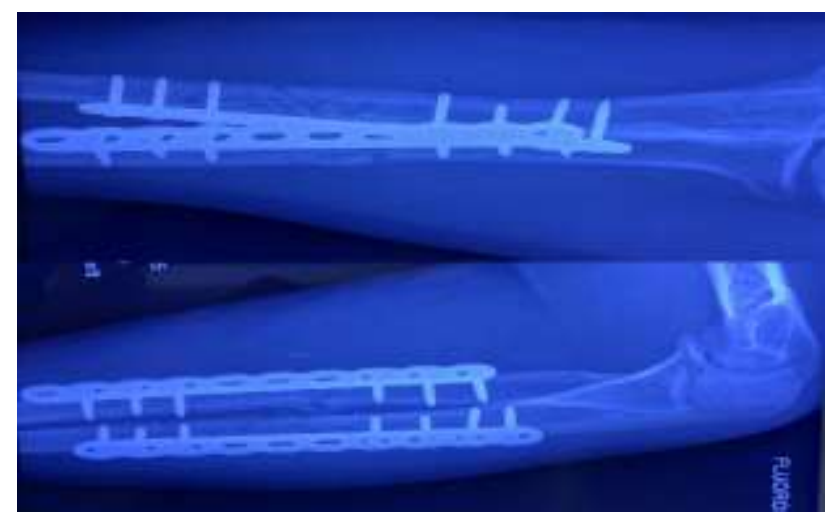

Figure 6: Post-operative radiograph after second surgery.

\section{DISCUSSION}

Both bone forearm fractures are relatively common fractures especially in developing countries. Owing to its excellent results, ORIF with compression plating has become a standard of care. ${ }^{3}$ Multiple complications including infection, wound dehiscence, nonunion, iatrogenic nerve injuries, radioulnar synostosis and refracture are known to perplex this procedure, with aseptic nonunion being reported in about $2-10 \%$ of the cases across literature. ${ }^{4}$ However, data regarding incidence of either ulna or radius fracture to end up in nonunion remains limited. Furthermore, despite all the advances among plating techniques and implant designs, controversies continue to exist regarding: need for primary bone grafting in acute comminuted fractures; type and length of plate and; refracture risks after plate removal. ${ }^{5,6}$ With refracture rates approaching $18 \%$, this less talked about complication occurs either through the original fracture site or through one of the empty screw holes after plate removal. ${ }^{7,8}$

Ways to prevent refractures after implant removal include: delayed hardware removal and splint or brace for protection. Fractures through screw holes have been reported to occur, even several months after the hardware removal surgery. $4.5 \mathrm{~mm}$ plates and screws are associated with increased propensity of refractures (up to 22\%), whereas $3.5 \mathrm{~mm}$ plating systems have reportedly less refracture rates. ${ }^{9}$
Our case depicts a dual failure mechanism. Due to trivial fall and impending non-union, the radius angulated at the original fracture site with the plate in situ. Instead of implant breakage, the good quality and better modulus of elasticity of the titanium implant rather led to plate bending and subsequent impaction at the fracture site. The failure of the plate by bending from the fracture site rather than breaking or loosening from one of the screw holes, emphasizes that the bone-implant construct had good biomechanical stability. Stable implant construct combined with the inability of the soft callus to bear the angular forces lead to failure at the fracture site with radius losing the race to union. The angulation at the radius fracture site, led to simultaneous forces on the ulna, but as the ulnar fracture had healed via primary bone union the plate rather failed from the proximal most screw site, a certain source of stress riser.

This case sheds light on the multiple technical issues in the primary and the revision surgery. First, the difference in rates of union among radius and ulna indicate either a lack of adequate biomechanical stability or increased soft tissue stripping (biological insult) at the radius fracture site compared to the ulna, since both the fractures had similar characteristics in per operative radiographs to begin with.

In the revision setting, in an attempt to minimize the stress risers and bridge the predrilled holes the ulnar plate was applied on the dorsal surface to avoid multiple drill holes on the previously used medial surface. However, due to technical challenges the radial plate was reapplied on the volar surface itself. Unfortunately, while working on the dorsal ulnar surface in the revision surgery, although the surgeons applied a longer plate, but the plate ended juxtaposed to the last screw hole of the previous surgery distally, highlighting the need for proper preoperative planning. Furthermore, extensive soft tissue dissection and pre-existent fibrosis led to either FPL tendon injury or neuropraxia of the anterior interosseous nerve begetting inability to flex the first interphalangeal joint, indicative of loss of FPL. Fortunately, the patient regained FPL function about 6months postoperatively, signifying physiological reactivation of AIN function or FPL tendon recovery via fibrosis. This rare but already described phenomenon warrants watchful dissection and necessary preoperative counseling of the patient about this possible complication. At the six month follow up, while the ulna again united via primary bone healing, the radius fracture still demonstrates hypertrophic callus suggestive of impending union. The possible reasons for delayed union at the radius fracture site includes: refracture from the original fracture site, extensive periosteal stripping while operating on the same surface twice and lack of compression at the fracture site as bridge plating as employed in the revision surgery to address fracture comminution.

With no consensus, the decision regarding optimal timing for implant removal remains contentious for orthopedic surgeons around the globe. This case report further highlights an unusual refracture pattern in a seemingly 
fully functional and healing fracture, without implant failure. Gradual weakening of the bones subsequent to a rigid implant construct has been attributed to 'stress shielding'. Although six months interval is inadequate for the stress shielding to mitigate bone strength, but this unusual presentation features a case wherein the titanium implant molded and overwhelmed the bony strength causing bone to fracture rather than implant breakage. This emphasizes the need for orthopaedic surgeons to consider the deleterious effects of stress shielding while reoperating on fractured bones. Although autologous bone grafting was not deemed necessary intraoperatively in this case, the surgeons are always advised to prepare the donor graft site accordingly and informed consent for the same should be documented in conjunction with the patient. Although rare, this case underscores that with proper preoperative planning, appropriate patient counseling, and sound surgical technique and religiously following $\mathrm{AO}$ principles for fracture fixation; good results can be ensured in an atypical refracture pattern too.

\section{CONCLUSION}

Although uncommon, management of both bone forearm re-fractures remains challenging both for the patient as well as the surgeon. Risk factors for nonunion or delayed union for either bone remains debatable. Re-ORIF with plating constitutes the gold standard of care. This case report discusses one such unusual case, and elaborates the importance of appropriate preoperative planning to ensure spanning of all previous screw holes, being prepared for autologous bone grafting, careful surgical dissection, thorough patient counseling including outcomes and expectations, planning implant removal cautiously after affirming union radiologically and expecting unusual complications like AIN palsy, tendon injury or delayed union. Ensuing aforementioned strategies may help achieve good clinical, functional and radiological outcome despite an oddball fracture pattern.

Funding: No funding sources Conflict of interest: None declared

Ethical approval: Not required

\section{REFERENCES}

1. Tornetta P, Ricci W, Court-Brown CM, Heckman JD, McQueen MM, McKee M. Rockwood and Green's fractures in adults. Lippincott Williams and Wilkins. 2015.

2. Keogh P, Khan H, Cooke E, Mccoy G. Loss of Flexor Pollicis Longus Function after Plating of the Radius. J Hand Surg Am. 1997;22:375-6.

3. Anderson LD, Sisk TD, Tooms RE, Park WI. Compression plate fixation in acute diaphyseal fractures of the radius and ulna. J Bone Jt Surg. 1975;57:287-97.

4. Boussakri H, Elibrahimi A, Bachiri M. Nonunion of fractures of the ulna and radius diaphyses: Clinical and radiological results of surgical treatment. Malaysian Orthop J. 2016;10:27-34.

5. Kloen P, Wiggers JK, Buijze GA. Treatment of diaphyseal non-unions of the ulna and radius. Arch Orthop Trauma Surg. 2010;130:1439-45.

6. Rosson JW, Shearer JR. Refracture after the removal of plates from the forearm. An avoidable complication. J Bone Jt Surg. 1991;73:415-7.

7. Brooks DB, Burstein AH, Frankel VH. The biomechanics of torsional fractures. The stress concentration effect of a drill hole. J Bone Joint Surg Am. 1070;52:507-14.

8. Cheal EJ, Hayes WC, White AA, Perren SM. Stress analysis of compression plate fixation and its effects on long bone remodeling. J Biomech. 1985;18:14150 .

9. Chapman MW, Gordon E, Zissimos AG. Compression-plate fixation of acute fractures of the diaphyses of the radius and ulna. J Bone Jt Surg. 1989;71:159-69.

Cite this article as: Vatsya P, Gupta A, Mittal S, Trikha V. Bone versus implant: an atypical presentation of a typical complication of forearm fractures. Int J Res Orthop 2021;7:860-3. 